\title{
Comparación entre Interpoladores Espaciales en el Estudio de Distribución de Partículas Sedimentables Insolubles en la Cuenca Atmosférica de Lima y Callao
}

\author{
Luis R. Chirinos ${ }^{(1)}$ y Carmen G. Mallqui (2) \\ (1) Departamento de Ingeniería, Sección Ingeniería Mecánica, Pontificia Universidad Católica del Perú. \\ Avenida Universitaria, 1801, Lima - Perú (e-mail: Ichirin@pucp.edu.pe) \\ (2) Departamento de Humanidades, Sección de Geografía, Pontificia Universidad Católica del Perú. \\ Avenida Universitaria, 1801, Lima - Perú (e-mail: carmen.mallqui@pucp.pe)
}

Recibido Dic. 7, 2015; Aceptado Feb. 10, 2016; Versión final Feb. 29, 2016, Publicado Ago. 2016

\begin{abstract}
Resumen
El objetivo del trabajo fue representar la distribución espacial de las partículas sedimentables insolubles, en la cuenca atmosférica de Lima y Callao. Esto se hace con un interpolador espacial que reproduce superficies de distribución acorde con los registros de campo. Se utilizaron los registros de material, correspondientes a las estaciones de invierno y verano, en 33 puntos de muestreo (norma ASTM D-173998). Los registros gravimétricos del material insoluble fueron procesados con el software ArcGis para obtener las superficies de distribución mediante los interpoladores de pesos de distancia inversa (Inverse Distance Weighting, IDW), e interpoladores Kriging y Spline. Con el método Spline y Kriging se obtuvieron rangos de valores negativos para la masa y rangos superiores a los valores de campo, respectivamente. Con el método IDW, los registros fueron similares a los obtenidos en campo. Basado en los resultados, se concluye que el método de interpolación IDW representa las superficies de distribución del material insoluble sedimentable en la cuenca atmosférica estudiada.
\end{abstract}

\section{Comparison between Spatial Interpolators in the Study of the Distribution of Non-Soluble Sediment Particles in the Atmospheric Basin of Lima and Callao}

\begin{abstract}
The objective of this research was to represent the spatial distribution of non-soluble sediment particles in the atmospheric basin of Lima and Callao. For this, a spatial interpolator that reproduces the surface distribution close to field values is used. Material collected during winter and summer in 33 sampling points was used (ASTM D-1739-98 norm). Gravimetric registers of non-soluble material were processed with ArcGIS software to obtain the surface distribution by using spatial interpolation methods known as Inverse Distance Weighting (IDW), and also the methods called Kriging and Spline. With the spline method, ranges of negative values for mass weight were obtained. With the Kriging method, the mass values were superior to those obtained in the field. In the case of the IDW method, the values were similar to those obtained in field work. Based on the results, the IDW method represents the best surface distribution method for non-soluble sediment particles in the atmospheric basin.
\end{abstract}




\section{INTRODUCCIÓN}

El análisis espacial se define como "una colección de técnicas y modelos que explícitamente usan referencias espaciales asociadas a cada valor de la variable que está especificada dentro del sistema que se está estudiando" (Haining, et al., 2010); es decir, que a través del tratamiento de información que tiene una referencia espacial ya dada se pueden obtener datos generales del sistema materia de estudio en el que se encuentra esta información. Asimismo, Sánchez y Amorós (2012) añaden que el análisis espacial se encarga de los "estudios de la estructura y relaciones territoriales a partir del conocimiento de la posición de las entidades geográficas y las características de las variables involucradas". A partir de la información obtenida por el análisis espacial se puede generar modelos espaciales, que describen los patrones de comportamiento de las variables estudiadas.

Dentro del campo de la geografía, el uso de Sistemas de Información Geográfica (siglas en inglés GIS) han contribuido al análisis espacial simplificando procesos y mejorando la precisión de las predicciones que se pueden generar a partir del estudio de determinadas variables y, de esta manera, se genera una mejor visualización de la distribución espacial de las variables. Los datos tratados con el análisis espacial pueden ser de tres tipos: (i) datos puntuales, (ii) superficies continuas y (iii) áreas. Los datos puntuales pueden ser "cualquier tipo de fenómeno localizable en el espacio que está asociado a una representación puntual" (Câmara, et al., 2004). Como ejemplos de ello se tendría, la textura del suelo en un campo de cultivo medida en un punto específico dentro de una parcela (Gozdowski, et. al., 2015) o mediciones puntuales del cobre contenido en los suelos afectados por la actividad industrial (Gonzáles, et.al., 2014). Otro caso de estudio podría ser las partículas sedimentables insolubles recolectadas en diferentes puntos de un área de estudio.

Para el análisis de datos puntuales existen diversas técnicas de interpolación y la elección de una de ellas depende del objetivo del estudio; sin embargo, la técnica más adecuada para tal fin es la interpolación de la variable de estudio. Joaquín Bosque (2000) sostiene que la interpolación espacial es "un procedimiento que permite calcular el valor de una variable en una posición del espacio, conociendo los valores de esa variable en otras posiciones de dicho espacio"; es decir, a partir del conocimiento del valor de un punto podemos derivar el valor de los puntos que lo rodean y crear superficies continuas influenciadas por el valor del punto real existente. De esta forma, la interpolación de datos puntuales permite proyectar mapas o superficies continuas a partir de datos discretos (Shahbazi, et. al., 2015; Cruz-Cárdenas, et. al., 2010; Johnston et. al., 2001). Los interpoladores se pueden clasificar en determinísticos y geo-estadísticos. En el primer caso, estos interpoladores crean superficies basadas en las mediciones puntuales o en fórmulas matemáticas predeterminadas, mientras que los interpoladores geo-estadísticos se basan en estimaciones del parámetro de interés con procesos estadísticos, y sus resultados son usados para predicciones más detalladas de las superficies finales.

Los interpoladores más usados en los estudios de análisis espacial son los siguientes: (i) Función Inversa de la Distancia o "Inverse Distance Weighting", (siglas en inglés IDW), (ii) Método de Interpolación Kriging y (iii) Método de Interpolación Spline. No obstante, los resultados obtenidos utilizando los métodos mencionados, no siempre son los mismos, pues los fenómenos atmosféricos tienen particularidades, de acuerdo al espacio geográfico en el que ocurren, que deben ser tomadas en cuenta al momento de analizar los resultados obtenidos con los Sistemas de Información Geográfica. El método geo-estadístico más usado es el interpolador Kriging, el cual se basa en supuestos estadísticos y se emplean en predicciones avanzadas de superficies. Asimismo dicho interpolador asume que la distancia entre los puntos refleja una correlación espacial que se puede utilizar para explicar las variaciones de los valores en la superficie. El método funciona usando un número específico de puntos o todos los puntos dentro de un radio de cobertura para determinar el valor resultante para cada ubicación. Este método es más apropiado cuando la distancia es conocida y se utiliza para aplicaciones en la ciencia y la geología del suelo y la hidrología. Hay varios tipos de interpoladores Kriging, entre ellos se encuentran el interpolador Kriging Ordinario que es el método más común, y el interpolador Kriging Universal (Li, y Heap, 2008; Childs, 2004).

Por otro lado, los métodos de interpolación determinísticos se encargan de calcular un valor para una celda de la cuadrícula usando únicamente las propiedades físicas de las muestras de elevación; es decir, crea superficies basadas en los puntos medidos y en fórmulas matemáticas (Childs, 2004; Goovaerts, 1997). El método de interpolación determinístico más común es la ponderación en función inversa de la distancia o también conocido como IDW. Este método asume que "cada punto medido tiene una influencia local que disminuye con la distancia. Le asigna una ponderación mayor a los puntos más cercanos por predecir, que a aquellos que se encuentran más alejados, de ahí el nombre de distancia inversa ponderada" (Toro y Melo, 2009). 
Otro interpolador determinístico, no tan conocido como el IDW, es el interpolador Spline, el cual estima valores usando una función matemática que se encarga de minimizar la curvatura de la superficie total, en donde se ubican los datos puntuales para dar como resultado una superficie lisa que pasa exactamente a través de los puntos de entrada y reduce al mínimo la curvatura total de la superficie. El interpolador Spline se recomienda para estudios de caso donde las variables deriven de fenómenos como la temperatura ( $\mathrm{Li}$, y Heap, 2008; Childs, 2004). Los autores citados anteriormente no hacen referencia que el parámetro de estudio podría ser un contaminante en general, sin embargo en la literatura se identifican estudios realizados con estos métodos para generar mapas de distribución espacial de contaminantes identificando zonas de mayor y menor concentración (Nethaji Mariappan, et. al., 2013; Pearce, et. al., 2008).

El área de Lima Metropolitana y el Callao, se encuentra entre las más contaminadas de Sudamérica por partículas totales suspendidas y otros contaminantes, indicando como principal fuente de dicha contaminación al sector transporte, el sector industrial y el sector energético (Ministerio del Medio Ambiente - MINAM, 2012). Cabe señalar que dichos estudios no presentan la distribución espacial de dicha contaminación, por lo que se propone en el presente trabajo la determinación de la distribución espacial de las partículas sedimentables insolubles. Cabe señalar que dicho material representa a los contaminantes que no cambian su constitución con los procesos físicos o químicos que tienen lugar en la atmósfera.

Por ello, el objetivo de este estudio es la identificación del interpolador espacial más adecuado para el estudio de la distribución espacial de las partículas sedimentables insolubles en la cuenca atmosférica de Lima Metropolitana y el Callao. La elección del área de estudio está sometida a un proceso de conurbación soportado por un creciente desarrollo industrial, del parque automotor y de la infraestructura, cuyas actividades generan contaminantes atmosféricos, que forman parte de las partículas sedimentables que bajo procesos de sedimentación seca o húmeda alcanzan la superficie del área de estudio.

\section{MATERIALES Y MÉTODOS}

La sección materiales y métodos se divide en tres subsecciones: en la primera, se define el área de estudio; en la segunda, se explican los criterios adoptados para la ubicación de los puntos de muestreo y cómo se realiza la recolección de datos en cada estación de muestreo y, en la tercera parte se describe el manejo de los datos mediante el software ArcGis.

\section{Área de estudio}

La cuenca atmosférica de Lima y Callao es un espacio geográfico delimitado por barreras topográficas naturales como la línea costera, los acantilados y las lomas; así como también divisiones administrativas y políticas. Dentro de esta cuenca "se modifica la circulación general de la atmósfera sobre la superficie (capa límite de la atmósfera) dando lugar a la formación de un campo de vientos locales, diferentes del flujo de la atmósfera libre" (Servicio Nacional de Meteorología e Hidrología-SENAMHI, 2006). Estos vientos locales más la circulación de vientos en general son los que generan la suspensión de las partículas y los que se encargan de transportarlas y dispersarlas al interior de la cuenca.

Esta cuenca abarca todos los distritos de la provincia constitucional del Callao y también la mayoría de distritos de Lima Metropolitana. Estos se desarrollan sobre una planicie costera de $10 \mathrm{~km}$ de ancho en promedio y una altura comprendida entre los 0 a 200 m.s.n.m. A medida que la planicie costera se aleja del mar aparecen pequeñas lomas y pequeños cerros que delimitan la cuenca y crean condiciones meteorológicas particulares. En la actualidad, la población del área de estudio debe superar los 9450585 habitantes reportados el año 2012 (Instituto Nacional de Estadística e Informática - INEI, 2012).

La cuenca atmosférica de Lima y Callao es un espacio geográfico amplio por lo cual se necesitaría una gran cantidad de puntos de monitoreo de calidad del aire; sin embargo, eso no es factible por los costos y el esfuerzo que demandaría la recolección de la data en cada uno de ellos. Por ello, para poder conocer el área de influencia de cada punto es necesario realizar interpolaciones que nos permitan predecir el valor de los parámetros de interés en dichas superficies.

\section{Puntos de muestreo y recolección de datos}

Los 33 puntos de muestreo identificados en el área de estudio corresponden a estaciones de muestreo utilizadas por el SENAMHI como parte de su programa de monitoreo de partículas totales en suspensión. Los criterios de ubicación de los puntos fueron que el lugar de emplazamiento esté libre de obstáculos que limiten la circulación y que la dirección de las estaciones sigan una dirección de norte (N) a sur (S) y en la medida de lo posible deberían ubicarse en la parte central (SENAMHI, 2006). 
La siguiente etapa consistió en el recojo de los recipientes con el material sedimentable tomando en cuenta la propuesto en la Norma ASTM D-1739-98. El material depositado en cada recipiente se recupera con sucesivos lavados con agua micro-filtrada hasta alcanzar $200 \mathrm{ml}$. El concentrado obtenido se seca en estufa a $60^{\circ} \mathrm{C}$ hasta alcanzar un peso constante. El material insoluble se guarda en viales etiquetados con un ID (lugar, fecha de recolección, y estación de muestreo). Los datos fueron tomados en un periodo de 12 meses, 4 muestreos (uno por estación del año) en los 33 puntos de muestreo. El periodo de muestreo se inició el 21 de marzo del 2011 y finalizó el 20 de marzo del 2012, y los resultados presentados se hicieron considerando tan solo las muestras de invierno (2011) y verano (2012).

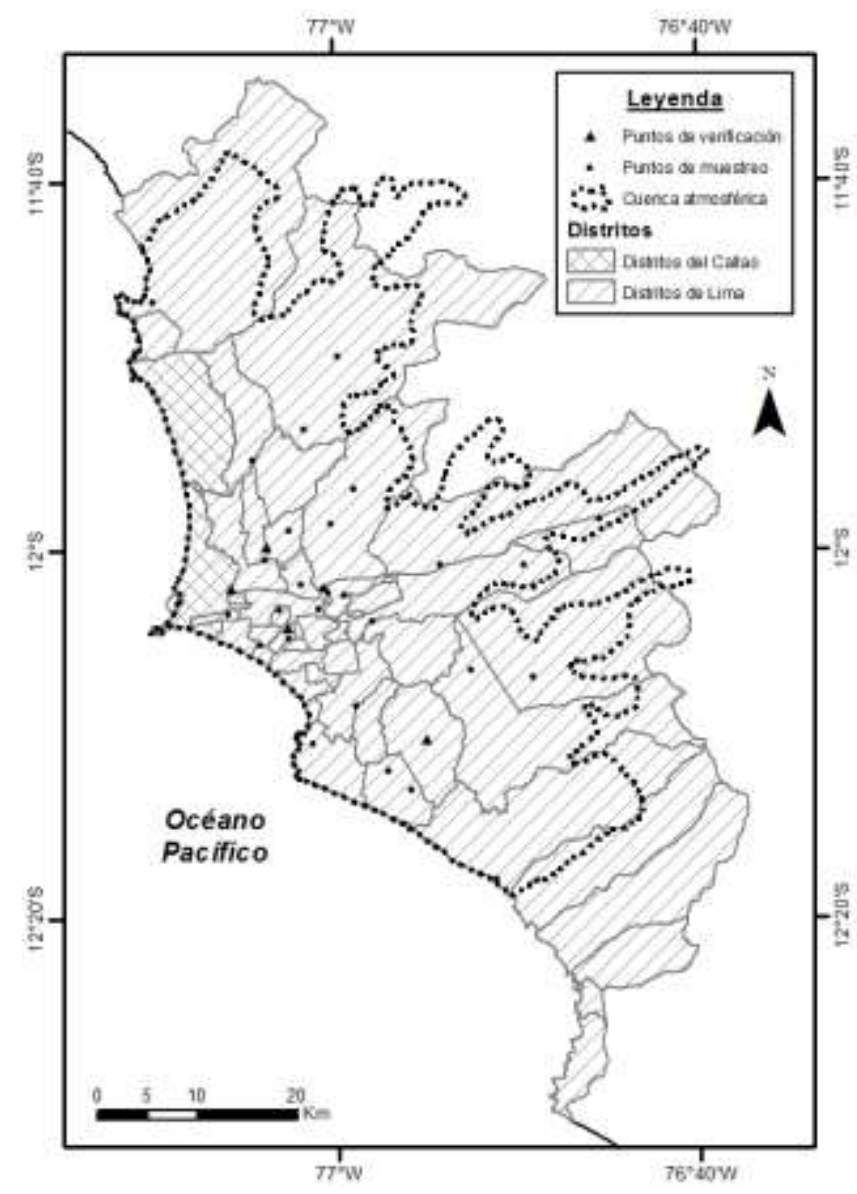

Fig.1: Ubicación de los puntos de muestreo en la cuenca atmosférica Lima - Callao

\section{Manejo de datos}

Con los datos obtenidos se elaboró una base de datos, que incluye un ID (identificación de cada punto de muestreo), nombre del punto de muestreo, coordenadas UTM (X, Y), la altitud de cada punto (m.s.n.m.) y el valor del punto por estación (gramos totales de material sedimentable insoluble), tal como se muestra en la Tabla 1. Antes de elaborar los mapas finales de interpolación, se eligieron cuatro puntos para validar el método que se pueden observar en la Fig.1 y que se describen en la Tabla 1. Además, en esta ocasión, solo se trabajaron con las estaciones de invierno (año: 2011) y verano (año: 2012), para observar cómo las condiciones climáticas opuestas influyen en la distribución espacial del material.

Los mapas de interpolación, se elaboraron con el programa ArcMap del software ArcGis 10.0. En este programa se abrió la base de datos que se preparó en Excel (como se muestra en la Tabla 1) y a través de la función Display $X Y$ Data se ingresó la información de las coordenadas (UTM) para obtener los puntos de muestreo con referencia espacial. Los puntos georeferenciados tienen una Tabla de Atributos que contiene la información de los pesos por estaciones, necesarios para generar las superficies de influencia con los interpoladores IDW, Spline y Kriging. Estos interpoladores se encuentran en la caja de herramientas de Spatial Analyst Tools dentro de la categoría Interpolation. Para el caso del interpolador Kriging, el programa opera según la siguiente ecuación:

$\hat{Z}\left(S_{0}\right)=\sum_{i=1}^{N} \lambda_{i} Z\left(S_{i}\right)$ 
Tabla 1: Registros material sedimentable insoluble total $(\mathrm{g})$ por punto de muestreo y según estación

\begin{tabular}{|c|c|c|c|c|c|c|}
\hline$N^{\circ}$ & Nombre & $x$ & Y & $\begin{array}{l}\text { Altitud }(\mathrm{H}) \\
\text { m.s.n.m. }\end{array}$ & Invierno & Verano \\
\hline 1 & Chaclacayo $\mathrm{Km} 26$ & 308725.00 & 8675895.33 & 708 & 20.5904 & 19.6128 \\
\hline 2 & $\begin{array}{l}\text { Mercado San Juan de } \\
\text { Lurigancho }\end{array}$ & 284189.13 & 8678929.52 & 379 & 20.8627 & 20.8535 \\
\hline 3 & $\begin{array}{l}\text { Municipalidad de } \\
\text { Carabayllo }\end{array}$ & 279220.60 & 8684783.62 & 234 & 21.4291 & 27.7006 \\
\hline 4 & $\begin{array}{l}\text { Las Flores Panamericana } \\
\text { Sur }\end{array}$ & 284453.26 & 8657115.43 & 122 & 25.4822 & 37.6193 \\
\hline 5 & Chorrillos & 280077.63 & 8653386.45 & 56 & 3.9617 & 4.2814 \\
\hline 6 & Manchay & 295920.41 & 8660694.92 & 401 & 17.1916 & 22.1187 \\
\hline 7 & Agua Azul Carabayllo & 282624.88 & 8692089.04 & 354 & 10.3062 & 20.1665 \\
\hline 8 & $\begin{array}{l}\text { Alcazar Rimac (Colegio } \\
\text { Leoncio Prado) }\end{array}$ & 278974.85 & 8669313.14 & 163 & 6.3701 & 6.5510 \\
\hline 9 & $\begin{array}{l}\text { Villa Maria del Triunfo - } \\
\text { Cementos Lima. (Punto } \\
\text { de Validación) }\end{array}$ & 291488.37 & 8653665.18 & 272 & 31.7828 & 28.2067 \\
\hline 10 & Magdalena & 274837.78 & 8663178.46 & 88 & 3.5962 & 4.6999 \\
\hline 11 & $\begin{array}{l}\text { Campo de Marte } \\
\text { (Punto de Validación) }\end{array}$ & 277599.91 & 8664813.49 & 137 & 5.1805 & 4.0859 \\
\hline 12 & $\begin{array}{l}\text { Colegio Nicolas } \\
\text { Copernico - Av. Proceres } \\
\text { de la Independencia } \\
\end{array}$ & 281923.38 & 8675318.63 & 270 & 14.6717 & 16.2937 \\
\hline 13 & Lurin Km 34 P. Sur & 298459.33 & 8639828.4 & 31 & 12.1956 & 14.0394 \\
\hline 14 & $\begin{array}{l}\text { Barrios Altos (Colegio } \\
\text { Nstra. Sr. De la } \\
\text { Misericordia) }\end{array}$ & 280671.33 & 8666872.76 & 201 & 6.7118 & 7.5999 \\
\hline 15 & $\begin{array}{l}\text { Villa Salvador Ins. } \\
\text { Gonzales Prada }\end{array}$ & 289956.97 & 8648783.65 & 215 & 6.3938 & 13.6301 \\
\hline 16 & Separadora Industrial & 286051.27 & 8665565.49 & 257 & 15.3368 & 16.3144 \\
\hline 17 & $\begin{array}{l}\text { Quilca } \\
\text { (Punto de Validación) }\end{array}$ & 271856.77 & 8668747.59 & 61 & 16.6397 & 17.0125 \\
\hline 18 & Senamhi & 277678.27 & 8663849.45 & 136 & 6.4066 & 5.2828 \\
\hline 19 & Hospital H. Unanue & 283193.04 & 8668217.86 & 241 & 5.4893 & 7.9251 \\
\hline 20 & Mepsa III & 281061.54 & 8668805.27 & 203 & 28.0733 & 23.2071 \\
\hline 21 & Faucett & 271613.28 & 8666327.28 & 64 & 5.1330 & 5.7981 \\
\hline 22 & Cieneguilla & 302145.63 & 8660090.66 & 319 & 11.9416 & 14.1210 \\
\hline 23 & Mepsa & 281645.60 & 8668479.64 & 227 & 69.4204 & 66.8219 \\
\hline 24 & Huaycan Radio Enmanuel & 301272.87 & 8671261.63 & 583 & 17.4309 & 18.2331 \\
\hline 25 & $\begin{array}{l}\text { Tomas Catari Colegio } \\
\text { Quiñones }\end{array}$ & 275291.39 & 8671747.75 & 103 & 14.2495 & 19.7425 \\
\hline 26 & Huachipa & 292833.10 & 8671355.94 & 399 & 98.3928 & 66.0563 \\
\hline 27 & $\begin{array}{l}\text { El Mirador Independencia } \\
\text { III } \\
\text { (Punto de Validación) }\end{array}$ & 275407.93 & 8672967.39 & 88 & 15.6986 & 18.2788 \\
\hline 28 & Evitamiento Mepsa II & 281235.03 & 8668890.38 & 203 & 35.2511 & 32.2689 \\
\hline 29 & $\begin{array}{l}\text { Villa Salvador I (Sector J, } \\
\text { Grupo } 19 \mathrm{Mz} . \mathrm{M} \text { - Zona B) } \\
\end{array}$ & 287704.65 & 8650663.54 & 164 & 30.5804 & 30.2533 \\
\hline 30 & Santa Rosa Ancon & 264107.00 & 8697494.92 & 25 & 3.9141 & 7.1430 \\
\hline 31 & Puente Chillón & 274095.02 & 8681761.05 & 136 & 11.7388 & 24.0861 \\
\hline 32 & Breña & 276751.71 & 8666638.68 & 139 & 7.3897 & 6.9439 \\
\hline 33 & $\begin{array}{l}\text { Coricancha } \\
\text { Independencia }\end{array}$ & 277744.73 & 8674727.67 & 207 & 11.6511 & 11.6511 \\
\hline
\end{tabular}

En esta ecuación se pondera los valores medidos en determinadas ubicaciones para calcular una predicción de las ubicaciones sin mediciones; es decir, las superficies de influencia. Para que esta condición se cumpla $Z\left(S_{i}\right)$ es el valor medido de la ubicación $i, \lambda_{i}$ es una ponderación desconocida para el valor medido en la ubicación $i, S_{0}$ es la ubicación de la predicción y $N$ es la cantidad de valores medidos.

Para operar con éxito la ecuación del interpolador Kriging, el programa ArcGis solicita un Input point feature que son los puntos de muestreo georeferenciados y el $Z$ Value Field que corresponde al peso de la muestra por cada estación contenida en la Tabla de Atributos de los puntos de muestreo. Entre los dos métodos que se ofrecen se elige el método de Kriging Ordinario porque al momento de realizar la ponderación, $\lambda_{i}$, el 
modelo debe ser ajustado a tres variables: los puntos medidos, la distancia a la ubicación de la predicción y las relaciones espaciales entre los valores medidos alrededor de la ubicación de la predicción. Por lo tanto, se ajusta a las condiciones previas definidas para la interpolación. Los otros parámetros solicitados son opcionales y los valores considerados corresponden a la condición base o también conocida como default.

En el caso del interpolador IDW, el programa opera con la misma ecuación empleada para el interpolador Kriging pero en este caso la variable en cuestión es solamente la distancia a la ubicación de la predicción con respecto a la ponderación, $\lambda_{i}$. Al igual que el Kriging, el programa solicita un Input point feature y un $Z$ Value Field que fueron los mismos que se usaron en el caso del Kriging. Para el interpolador Spline, el programa usa la siguiente ecuación:

$S(x, y)=T(x, y)+\sum_{j=i}^{N} \lambda \mathrm{j} \mathrm{R}\left(r_{j}\right)$

En esta ecuación $j$ toma desde 1 hasta $N$; $N$ es la cantidad de puntos, $\lambda_{\mathrm{j}}$ son coeficientes determinados por la solución de un sistema de ecuaciones lineales y $r_{j}$ es la distancia del punto $(x, y)$ al punto $j$. Como se utilizará la opción Regularized, $\mathrm{T}(\mathrm{x}, \mathrm{y})=\mathrm{a}_{1}+\mathrm{a}_{2} \mathrm{x}+\mathrm{a}_{3} \mathrm{y}$; donde $\mathrm{a}_{i}$ son coeficientes determinados por la solución de un sistema de ecuaciones lineales. El programa para operar esta ecuación solicita los mismos datos que para el Kriging y el IDW.

\section{RESULTADOS Y DISCUSIÓN}

Los resultados obtenidos en cada punto de muestreo, según los métodos de interpolación Spline y Kriging se pueden ver en las Fig. 2 y 3 (mapas de distribución) en donde los valores representados para cada estación están expresados en $\mathrm{g} / \mathrm{m}^{2}$. En la Fig. 2 se puede observar las superficies obtenidas con el interpolador Kriging. Las superficies muestran variaciones poligonales que se representan en rangos con formas de mosaicos. Los valores generados para la masa de partículas sedimentables corresponden a rangos desde 9.23 hasta $31.57 \mathrm{~g} / \mathrm{m}^{2}$ y 10.9 a $29.96 \mathrm{~g} / \mathrm{m}^{2}$, en la estación de invierno y verano, respectivamente. En ambos casos los rangos no corresponden a los registros de campo.

En la Fig. 3 se observa que las zonas generadas con el interpolador Spline presentan superficies más suavizadas que en el caso anterior, sin embargo se obtienen valores negativos para el parámetro de estudio (masa registrada en $\mathrm{g} / \mathrm{m}^{2}$ ), condición inaceptable para el estudio propuesto (Ver Tabla 2). En la Fig. 4 se puede apreciar las superficies obtenidas con el método IDW con 29 datos puntuales, y conservando cuatro registros de campo para las verificaciones del caso. Los puntos de verificación están indicados en la Tabla 2 y corresponden a la Zona Norte, Zona Sur, Zona Este y Zona Oeste del área de estudio.

Tabla 2: Comparación de los valores mínimos y máximos entre interpoladores Spline, Kriging y el valor real

\begin{tabular}{|c|c|c|c|c|c|c|}
\hline \multirow{2}{*}{$\begin{array}{c}\text { Estación } \\
\text { del año }\end{array}$} & \multicolumn{2}{|c|}{ Valor Real } & \multicolumn{2}{c|}{ Spline } & \multicolumn{2}{c|}{ Kriging } \\
\cline { 2 - 7 } & $\begin{array}{c}\text { Valor Mínimo } \\
\left(\mathrm{g} / \mathrm{m}^{2}\right)\end{array}$ & $\begin{array}{c}\text { Valor Máximo } \\
\left(\mathrm{g} / \mathrm{m}^{2}\right)\end{array}$ & $\begin{array}{c}\text { Valor Mínimo } \\
\left(\mathrm{g} / \mathrm{m}^{2}\right)\end{array}$ & $\begin{array}{c}\text { Valor Máximo } \\
\left(\mathrm{g} / \mathrm{m}^{2}\right)\end{array}$ & $\begin{array}{c}\text { Valor Mínimo } \\
\left(\mathrm{g} / \mathrm{m}^{2}\right)\end{array}$ & $\begin{array}{c}\text { Valor Máximo } \\
\left(\mathrm{g} / \mathrm{m}^{2}\right)\end{array}$ \\
\hline Invierno & 3.5962 & 98.3928 & -80.93 & 136.06 & 9.23 & 31.57 \\
\hline Verano & 4.0859 & 66.8219 & -83.25 & 102.38 & 10.9 & 29.96 \\
\hline
\end{tabular}

Tabla 3: Comparación de los valores mínimos y máximos entre interpoladores IDW con 29 y 33 datos puntuales

\begin{tabular}{|c|c|c|c|c|c|c|}
\hline \multirow{2}{*}{$\begin{array}{c}\text { Estación } \\
\text { del año }\end{array}$} & \multicolumn{2}{|c|}{ Valor Real } & \multicolumn{2}{c|}{ IDW } & \multicolumn{2}{c|}{ IDW (29 puntos) } \\
\cline { 2 - 7 } & $\begin{array}{c}\text { Valor Mínimo } \\
\left(\mathrm{g} / \mathrm{m}^{2}\right)\end{array}$ & $\begin{array}{c}\text { Valor Máximo } \\
\left(\mathrm{g} / \mathrm{m}^{2}\right)\end{array}$ & $\begin{array}{c}\text { Valor Mínimo } \\
\left(\mathrm{g} / \mathrm{m}^{2}\right)\end{array}$ & $\begin{array}{c}\text { Valor Máximo } \\
\left(\mathrm{g} / \mathrm{m}^{2}\right)\end{array}$ & $\begin{array}{c}\text { Valor Mínimo } \\
\left(\mathrm{g} / \mathrm{m}^{2}\right)\end{array}$ & $\begin{array}{c}\text { Valor Máximo } \\
\left(\mathrm{g} / \mathrm{m}^{2}\right)\end{array}$ \\
\hline Invierno & 3.5962 & 98.3928 & 3.6 & 97.99 & 3.59 & 98.36 \\
\hline Verano & 4.0859 & 66.8219 & 4.11 & 66.04 & 4.29 & 66.04 \\
\hline
\end{tabular}

Este interpolador determinístico IDW, al igual que el interpolador Spline, genera superficies suavizadas en el área de estudio, siendo su principal diferencia la generación de puntos de concentración de partículas. Asimismo, este método genera valores mínimos y máximos del parámetro de estudio del mismo orden de magnitud que los valores registrados (Ver Tabla 3). Para validar el resultado obtenido se realizaron comparaciones de los valores registrados en campo, para los puntos $9(\bullet), 11(\star), 17(\boldsymbol{\bullet})$, y $27(\boldsymbol{\bullet})$; con las superficies generadas con el método IDW (Ver Fig. 4). De los cuatro puntos de validación, dos de ellos (11, 27) ratifican que la superficie generada refleja la realidad, mientras que los otros dos puntos 9 y 17 presentan un error de $9 \%$ y $36 \%$, respectivamente. 

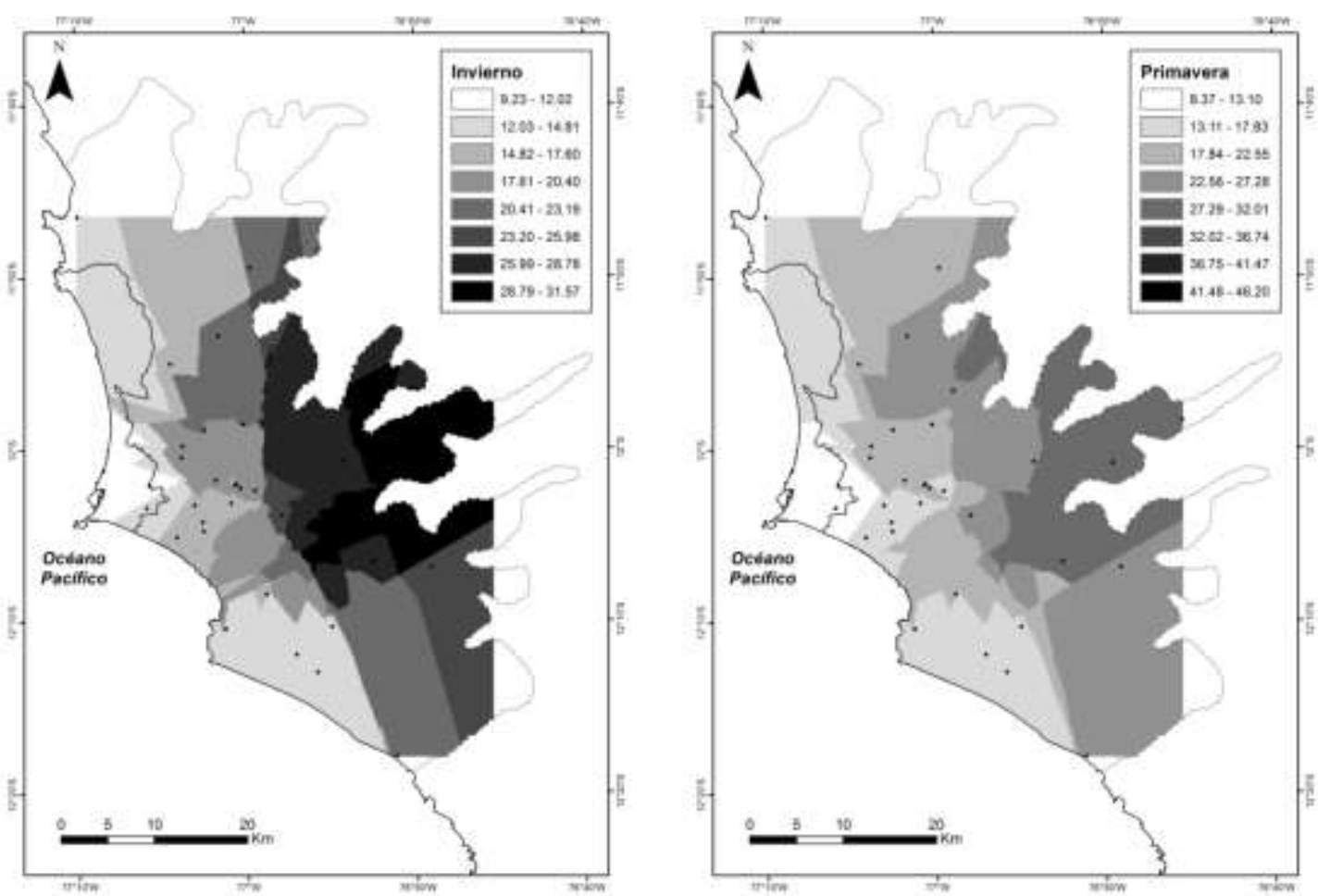

Fig. 2: Mapas con el método Interpolador Kriging
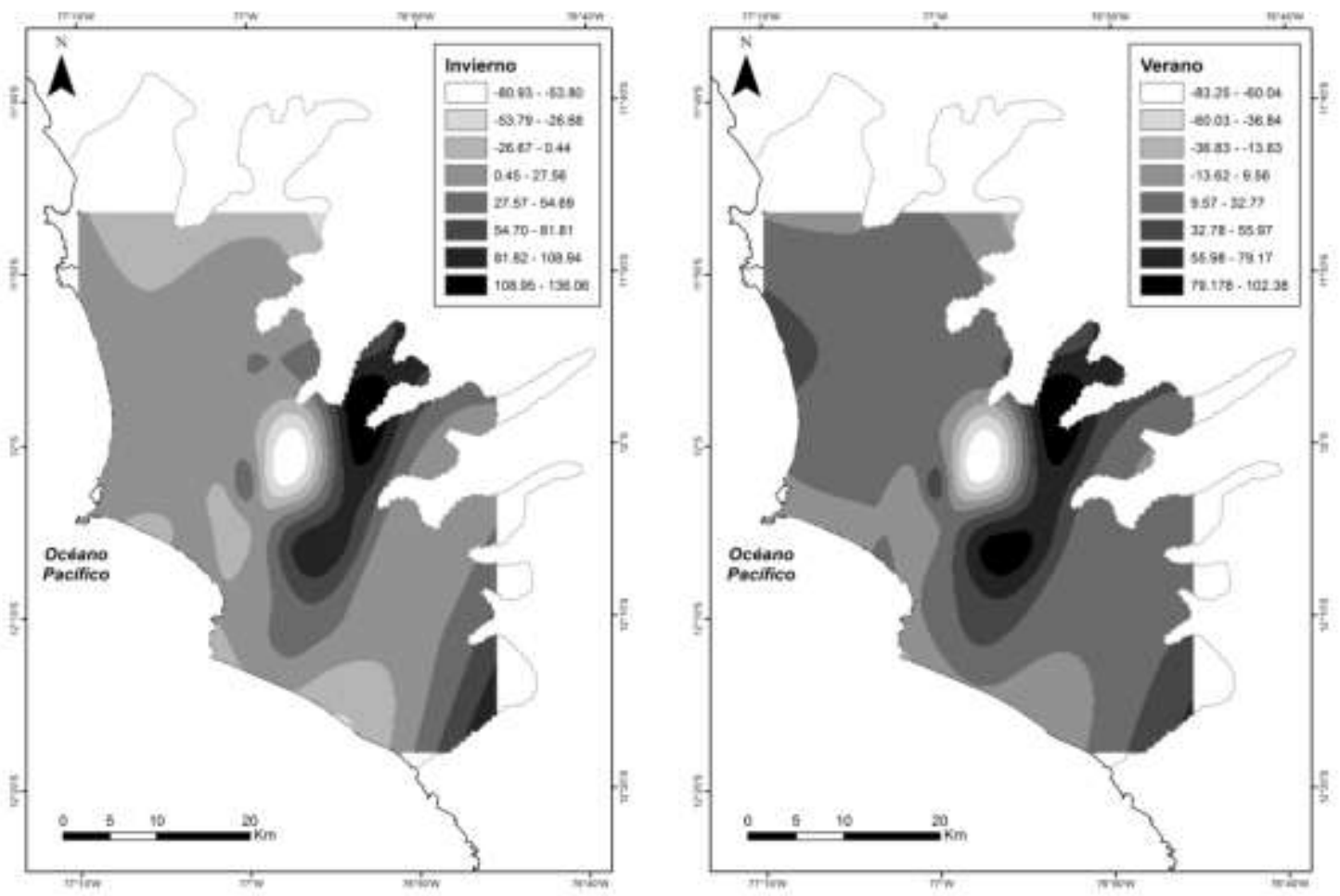

Fig. 3: Mapas con el método Interpolador Spline

Con el resultado anterior se pasa a la construcción de los mapas de distribución de partículas sedimentables insolubles con los 33 datos puntuales para la estaciones de verano e invierno del periodo de estudio (Ver Figura 5). Comparando los resultados de la Figura 4 y Figura 5, se observó que se conservan los rangos (zonas más oscuras). Para la estación de invierno los valores mínimo y máximo casi no han sufrido variación alguna, tan solo el nuevo valor mínimo se redujo en $0.01 \mathrm{~g} / \mathrm{m}^{2}$, y el valor máximo se incrementó en $0.37 \mathrm{~g} / \mathrm{m}^{2}$. Los puntos de máxima concentración en esta estación del año, se han mantenido en la Zona Centro y la Zona Este del área de estudio. En el caso de la Zona Oeste, la franja costera sigue manteniendo los valores más bajos, con un ligero aumento hacia el segundo rango de valores en la zona noroeste. 

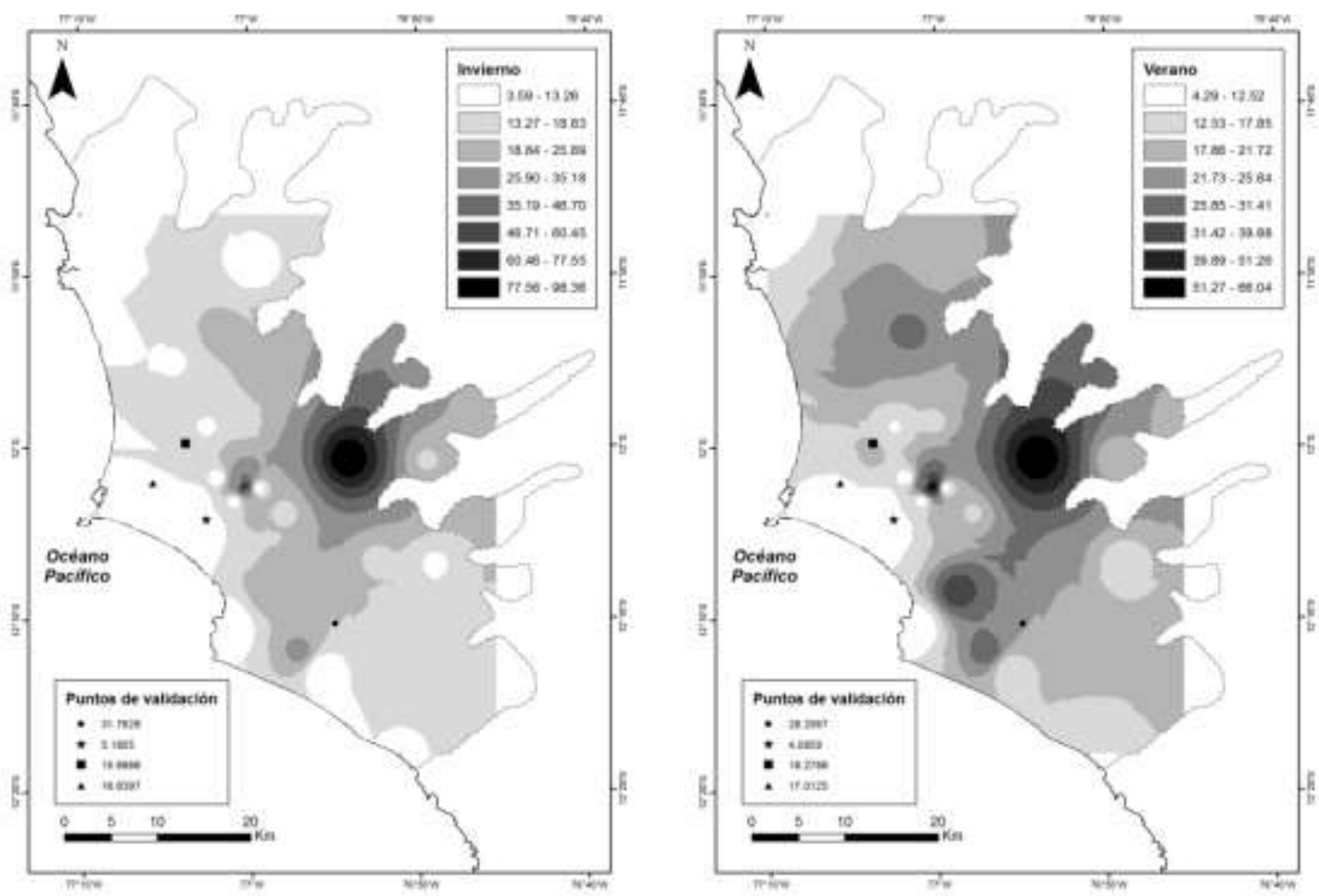

Fig. 4: Mapas con el método Interpolador IDW
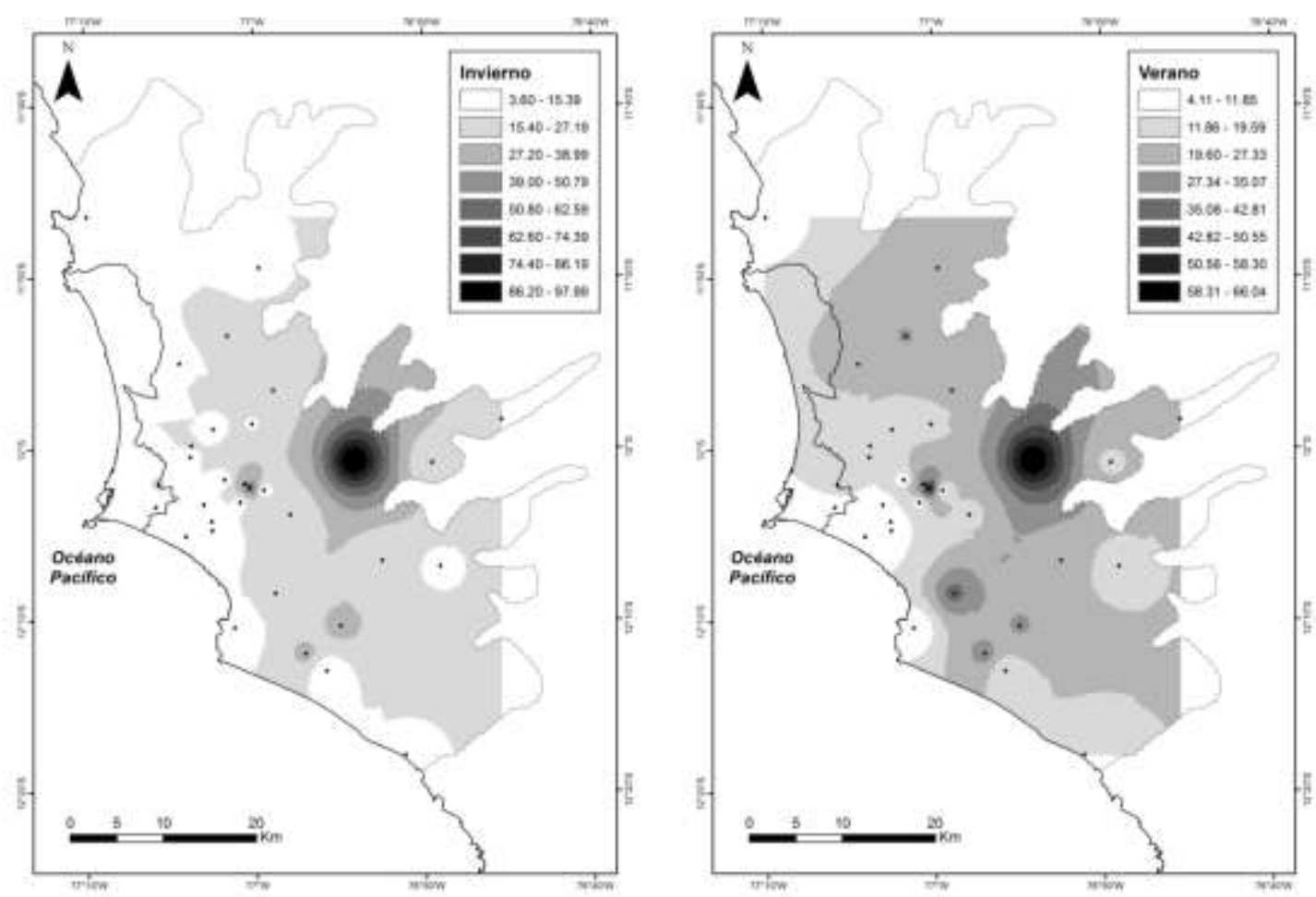

Fig. 5: Verificación del IDW

Los registros en la estación verano no presentan una marcada diferencia. El mínimo registro se incrementa en $0.18 \mathrm{~g} / \mathrm{m}^{2}$ y el máximo registro se mantiene en $66.4 \mathrm{~g} / \mathrm{m}^{2}$. En cuanto a la distribución de partículas, los registros de mayor concentración se mantuvieron en los mismos niveles, pero cabe mencionar que hacia la zona sur se identificaron tres zonas de alta concentración (mapa con 33 puntos) que no se generaron en el mapa con 29 puntos, sin embargo los valores se mantuvieron dentro del rango $50-60 \mathrm{~g} / \mathrm{m}^{2}$. En la Zona Noroeste las concentraciones se incrementaron pero sin sobrepasar los $17 \mathrm{~g} / \mathrm{m}^{2}$. 


\section{CONCLUSIONES}

En base a los resultados obtenidos, el análisis y la discusión, se puede concluir que los interpoladores Spline y Kriging utilizados con el programa ArcGis, permiten la generación de superficies de distribución a partir de datos puntuales del material sedimentable, sin embargo los rangos de abundancia no corresponden a los valores registrados. La selección del interpolador IDW dependió de la verificación de los rangos, los valores mínimos y máximos de la concentración de partículas y de la validación de los resultados con datos puntuales de campo (puntos 9, 11, 17 y 27) para la cuenca atmosférica de Lima Metropolitana y el Callao.

Los resultados permiten la identificación de zonas de mayor y menor concentración de partículas sedimentables, que para el caso de estudio corresponden a la Zona Este de Lima, que comprenden los distritos de Ate-Vitarte, San Juan de Lurigancho, entre otros. Por lo tanto, la generación de mapas de distribución espacial del material sedimentable para las otras estaciones del año, serían de utilidad para la toma de decisiones en la planificación urbana.

\section{AGRADECIMIENTOS}

Se agradece al Dr. Odón Sanchez, Director de Proyectos de Desarrollo y Medio Ambiente en Servicio Nacional de Meteorología e Hidrología del Perú (SENAMHI) y a la Dirección de Gestión de la Investigación de la PUCP por financiar el Proyecto de Investigación DGI-PUCP-2010-200.

\section{REFERENCIAS}

Bosque J., Sistemas de Información Geográfica, 2ª edición, pp. 451, Ediciones Rialp, Madrid, España (1997)

Câmara, G., Monteiro, A.M.V., Druck, S. y Carvalho, M.S., Análise Espacial e Geoprocessamento, en: Análise Espacial de Dados Geográficos de Druck, S; Carvalho, M.S.; Câmara, G. y Monteiro, A.V.M. (eds), EMBRAPA, Brasilia, Brasil (2004)

Childs, C., Interpolating Surfaces in ArcGis Spatial Analyst, (en la web:

https://www.esri.com/news/arcuser/0704/files/interpolating.pdf, acceso: 10 de julio, 2015), (2004)

Cruz-Cárdena, G., Ortiz-Solorio, C.A., Ojeda-Trejo, E., Martínez-Montoya, J.F., Sotel-Ruiz, E.D. y LiconaVargas, A.L., Digital Mapping of farmland and classes in three landscape in Mexico, doi: 10.4067/S071895162010000200003, Journal of soil science and plant nutrition, 10 (4), 414-427 (2010)

González, I., Neaman, A., Rubio, P. y Cortés, A., Spatial distribution of copper and pH in soils affected by intensive industrial activities in Puchuncaví and Quintero, central Chile, doi: 10.4067/S071895162014005000074, Journal of soil science and plant nutrition, 14 (4), 943-953 (2014)

Goovaerts, P., Geostatistics for Natural Resources Evaluation, pp. 483, Oxford University Press, New York, USA (1997)

Gozdowski, D., Stepién, M., Samborski, S., Dobers, E.S., Szatylowicz, J. y Chormanski, J., Prediction accuracy of selected spatial interpolation methods for soil texture at farm field scale, http://dx.doi.org/10.4067/S0718-95162015005000033, ISSN: 0718-9516, Journal of soil science and plant nutrition, 15 (3), 639-650 (2015)

Haining, R., Kerry, R. y Oliver, M. Geography, Spatial Data Analysis and Geostatistics, doi: 10.1111/j.15384632.2009.00780.x, Geographical Analysis, 42 (1), 7-31 (2010)

Instituto Nacional de Estadística e Información de Perú (INEI), 11 de julio. Día Mundial de la Población, (en la web: https://www.inei.gob.pe/media/MenuRecursivo/publicaciones_digitales/Est/Lib1032/libro.pdf, acceso: 10 de julio 2015), Lima, Perú (2012)

Johnston, K., Ver, Hoef, J.M., Krivoruchko, K. y Lucas, N., Using ArcGis Geostatistical Analyst, (en la web: http://dusk2.geo.orst.edu/gis/geostat_analyst.pdf, acceso: 20 de noviembre 2015), USA, Environmental Systems Research Institute. (ESRI) (2001)

Li, J. y Heap, A., A review of spatial interpolation methods for environmental scientists, (en la web: http://www.ga.gov.au/webtemp/image_cache/GA12526.pdf, acceso: 20 de octubre, 2015), Australian Government, Australia (2008) 
Ministerio del Ambiente (MINAM), Agenda Ambiente, Perú 2013 - 2014: Agenda Nacional de Acción Ambiental, (en la web: http://www.minam.gob.pe/wp-content/uploads/2013/06/agendambiental_peru_201320141.pdf, acceso: 20 de julio, 2015), Lima, Perú (2012)

Nethaji Mariappan, V., Mohana, P. y Murrugesan, C.A., Spatio-temporal assessment of air pollution from thermal stations and vehicular pollution from urban places in Tamil Nadu, http://sphinxsai.com/2013/janmar/chempdf/CT=27(172-179)JM13.pdf, ISSN: 0974-4290, Internacional Journal of ChemTech Research, 5 (1), 172 - 179 (2013)

Pearce, J., Rathbun, S., Aguilar-Villalobos, M. y Naeher, L., Characterizing the spatiotemporal variability of PM2.5 in Cusco, Perú using kriging with external drift, doi::10.1016/j.atmosenv.2008.10.060, Atmospheric Environment, 43, 2060-2069 (2009)

Sánchez, Y. y Amorós, M., Gestión del agua urbana mediante análisis espacial en los SIG, http://scielo.sld.cu/pdf/riha/v33n3/riha05312.pdf, ISSN: 1680-0338, Ingeniería Hidráulica, 33 (3), 58-71 (2012)

Servicio Nacional de Meteorología e Hidrología (SENAMHI), Evaluación de la Contaminación Atmosférica en la Zona Metropolitana de Lima-Callao/Junio 2006, (en la web:

http://www.senamhi.gob.pe/pdf/bol_cont_atmos0606.pdf, acceso: 20 de noviembre, 2015), Lima, Perú (2006)

Shahbazi, F., Aliasgharzad, N., Ebrahimzad, S.A. y Najafi, N., Geostatistical analysis for predicting soil biological maps under different scenarios of land use, doi:10.1016/j.ejsobi.2012.10.009, European Journal of Soil Biology, 55, 20-27 (2013)

Toro, G. y Melo, C., Aplicación de métodos de interpolación geoestadísticos para la predicción de niveles digitales de una imagen satelital con líneas perdidas y efecto sal y pimienta, http://www.redalyc.org/pdf/2570/257020606007.pdf, ISSN: 0123-921X, Tecnura, 12 (24), 57-69 (2009) 